\title{
Una prueba de rachas para la alternativa "estocásticamente mayor que"en muestras de la distribución lognormal ${ }^{1}$
}

\author{
A run test for the alternative "stochastically greater than "in samples \\ of the lognormal distribution
}

\author{
Jimmy A. Corzo S ${ }^{\text {a }}$ \\ jacorzos@unal.edu.co \\ Myrian E. Vergara Mb \\ mvergara@unisalle.edu.co
}

J. Giovany Babativa M.

josebabativa@usantotomas.edu.co

\begin{abstract}
Resumen
Se propone una prueba de rachas para la hipótesis de simetría alrededor de una mediana desconocida con alternativa de "estocósticamente mayor que" basada en una prueba de rachas recortada para la hipótesis de simetría alrededor de una mediana desconocida con alternativa de dos colas propuesta en Babativa \& Corzo (2010). Por medio de un estudio de simulación se encuentra que para muestras de la distribución lognormal la prueba propuesta mantiene su tamaño bajo la hipótesis de simetría, y que su potencia empírica supera la de las pruebas propuestas en Cabilio \& Masaro (1996), Mira (1999) y Miao et al. (2006).
\end{abstract}

Palabras clave: Pruebas de rachas recortadas, estocásticamente mayor que, potencia de una prueba.

\footnotetext{
Abstract

We propose a runs test for the hypothesis of symmetry around an unknown median with alternative "stochastically larger than" based on a trimmed runs test for the hypothesis of symmetry around an unknown median with two-tailed alternative proposed in Babativa \& Corzo (2010). By a simulation study we show that

${ }^{1}$ DOI: http://dx.doi.org/10.15332/s2027-3355.2017.0002.06

Corzo, J., Vergara, M. y Babativa, J. (2017) Una prueba de rachas para la alternativa "estocásticamente mayor que"en muestras de la distribución lognormal. Comunicaciones en Estadística, 10(2), $297-314$.

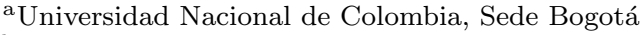

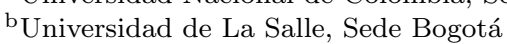

${ }^{\mathrm{c}}$ Universidad Santo Tomás, Sede Bogotá
} 
for samples coming from the Lognormal Distribution, the proposed test maintains the prefixed size and that its empirical power is larger than of the other compared tests proposed in Cabilio \& Masaro (1996), Mira (1999) and Miao et al. (2006).

Keywords: Trimmed runs tests, symmetry Tests, stochastically larger than, power of a test.

\section{Introducción}

El supuesto de simetría de la distribución muestreada es necesario en muchos de los métodos no paramétricos basados en rangos, para validar este supuesto existen varias pruebas con alternativa de dos colas conocidas también como "estocásticamente diferente". Por otra parte, en aplicaciones sobre la distribución del ingreso, el consumo o la sobrevivencia, resulta de sumo interés identificar la dirección de la asimetría. En el primer caso proporciona un indicador de inequidad; en el segundo puede utilizarse para identificar grandes consumidores potenciales, y en el caso de la sobrevivencia es útil para estimar la duración de algún aparato, o el tiempo que sobrevive un paciente después de un tratamiento. Para esto son necesarias pruebas con alternativa de cola derecha o "estocásticamente mayor"y de cola izquierda o "estocásticamente menor".

El tema se puede tratar como un problema de una muestra en el que se dispone de $N$ observaciones independientes $X_{1}^{\star}, \ldots, X_{N}^{\star}$ de una característica $X^{\star}$ provenientes de una distribución arbitraria continua $F(x-\theta)$ con mediana $\theta$. Para verificar si $F$ es simétrica se prueba la hipótesis:

$$
H_{0}: F(x-\theta)=1-F(\theta-x), \quad \forall x \in \mathbb{R}
$$

La asimetría se identifica con alguna de las siguientes alternativas:

$$
\begin{aligned}
& K_{1}: F(x-\theta) \neq 1-F(\theta-x), \text { para al menos un } x \in \mathbb{R} \text { (asimetría) } \\
& K_{2}: F(x-\theta)<1-F(\theta-x), \text { para al menos un } x \in \mathbb{R} \text { (estocásticamente mayor) } \\
& K_{3}: F(x-\theta)>1-F(\theta-x), \text { para al menos un } x \in \mathbb{R} \text { (estocásticamente menor) }
\end{aligned}
$$

Para las alternativas $K_{2}$ y $K_{3}$ se encuentran en la literatura las pruebas de Kolmogorov-Smirnov para una cola propuestas en Cabilio \& Masaro (1996), Mira (1999), Miao et al. (2006), Chatterjee \& Sen (1971) entre otras.

Las pruebas propuestas en Corzo \& Babativa (2013) para la alternativa de asimetría $K_{1}$ utilizan estadísticas de rachas recortadas, ponderadas positiva o negativamente según la cola en la que se encuentren las observaciones en la distribución muestreada, de manera que los signos de los valores de las estadísticas son indicadores de la cola para la cual se presenta la asimetría, este hecho se aprovechará para estudiar el comportamiento de la potencia empírica de dichas pruebas para 
la alternativa $K_{2}$. Para la alternativa $K_{3}$ se requieren cambios menores que se explican adelante. En la segunda sección, se introduce la prueba propuesta; en la tercera sección, se muestra el estudio de Monte Carlo y los resultados principales y en la cuarta sección, se da dan algunas conclusiones y recomendaciones.

\section{Prueba propuesta}

El número de rachas en la muestra se obtiene de la siguiente transformación: sea $\hat{\theta}$ la mediana muestral; $X_{i}=X_{i}^{\star}-\hat{\theta}, i=1, \ldots, N$ las observaciones centradas por la mediana muestral y $|X|_{(1)}, \ldots,|X|_{(N)}$ la sucesión ordenada de valores absolutos de las observaciones centradas. Se definen el rango $R_{i}$ de $\left|X_{i}\right|$ y el antirango $D_{j}$ de $|X|_{(j)}$ por las relaciones $\left|X_{i}\right|=|X|_{\left(R_{i}\right)}$ para $i=1, \ldots, N$ y $\left|X_{D_{j}}\right|=|X|_{(j)}$, para $j=1, \ldots, N$.

Se define la sucesión dicótoma

$$
\eta_{j}=\left\{\begin{array}{ll}
1 & \text { si } X_{D_{j}}>0 \\
0 & \text { en otro caso. }
\end{array} \quad j=1, \ldots, N .\right.
$$

En la cual están representadas las observaciones positivas por unos y las negativas por ceros. Aunque $\eta_{j}$ depende de $D_{j}$, por simplicidad solo se usará el subíndice $j$ teniendo en cuenta, cuando sea necesario, su dependencia de $D_{j}$. Dicha sucesión tiene la siguiente estructura:

$$
\begin{aligned}
\eta_{1}=\cdots=\eta_{L 1} \neq & \eta_{L_{1}+1}=\cdots=\eta_{L_{1}+L_{2}} \neq \eta_{L_{1}+L_{2}+1}=\cdots \\
\cdots & \neq \cdots=\eta_{L_{1}+\cdots+L_{S-1}} \neq \eta_{L_{1}+\cdots+L_{S-1}+1}=\cdots=\eta_{N}
\end{aligned}
$$

en la cual se distinguen $S \geq 1$ grupos de símbolos idénticos, cada uno de los cuales define una racha. Además, el $i$-ésimo grupo es de longitud $L_{i}$.

El número de rachas está determinado por los cambios en la sucesión dicótoma de observaciones positivas a negativas o viceversa. Para esto se define $I_{1}=1$ y los siguientes indicadores de cambios:

$$
I_{j}=\left\{\begin{array}{ll}
1 & \text { si } \eta_{j-1} \neq \eta_{j} \\
0 & \text { si } \eta_{j-1}=\eta_{j}
\end{array} \quad j=2, \ldots, N\right.
$$

Se define el número parcial de rachas hasta la $j$-ésima observación más pequeña así:

$$
S_{1}=1, \quad S_{j}=\sum_{k=1}^{j} I_{k}=1+\sum_{k=2}^{j} I_{k}, \quad j=2, \ldots, N
$$


En particular, $S_{N}=S$ es el número total de rachas en la sucesión dicótoma y corresponde a una estadística propuesta por McWilliams (1990) para la alternativa $K_{1}$.

Siguiendo a Modarres \& Gastwirth (1996) bajo $H_{0}$ se cumple $P\left(I_{k}=1\right)=P\left(I_{k}=\right.$ $0)=1 / 2$. En cambio, bajo $K_{2}, P\left(I_{k}=1\right) \geq P\left(I_{k}=0\right)$ y depende de $k$, debido a que la cola derecha de $F$ bajo $K_{2}$ es más larga que su cola izquierda. En estas condiciones ocurrirán muchas observaciones positivas en la cola derecha y por lo tanto pocas rachas de unos. Análogamente, bajo $K_{3}$ ocurrirán pocas rachas de ceros en la cola izquierda. Siguiendo la metodología propuesta por Corzo (1989) y tomando la idea de recortar observaciones de la muestra presentada por Modarres \& Gastwirth (1996) y Modarres \& Gastwirth (1998) se utiliza la estadística propuesta por Babativa \& Corzo (2010):

$$
R_{p}=\frac{1}{S_{N}} \sum_{i=[N p]+1}^{N} \phi\left(S_{i}, i\right) \delta_{i}
$$

donde

$$
\delta_{i}=\left\{\begin{array}{ll}
1 & \text { si } X_{D_{i}}>0 \\
-1 & \text { si } X_{D_{i}}<0
\end{array} \quad \text { para } i=1, \ldots, N\right.
$$

y

$$
\phi\left(S_{i}, i\right)= \begin{cases}S_{i}-p S_{N} & \text { si } i>[N p] \\ 0 & \text { en otro caso. }\end{cases}
$$

Aquí $p$ es una proporción de recorte; $[N p]$ es la parte entera de $N p$

Para identificar la región crítica, sea $x>0$ entonces bajo la alternativa $K_{2}$ se espera que $P(X \leq x)<P(X>-x)$ lo que implica que la muestra tendrá más observaciones positivas que negativas, generándose pocas rachas de unos con longitudes grandes en las últimas posiciones (valores grandes de $i$ ). Como son rachas largas de unos el valor de $\delta_{i}$ es positivo y por tanto los valores de $R_{p}$ tenderán a ser positivos y grandes, razón por la que la prueba rechaza $H_{0}$ a favor de $K_{2}$ cuando $R_{p}$ toma valores extremos positivos. Con un análisis análogo se puede concluir que la prueba rechaza $H_{0}$ a favor de $K_{3}$ cuando $R_{p}$ toma valores extremos negativos.

En Corzo \& Babativa (2013) se demuestra que $E\left(R_{p}\right)=0$ y que la varianza se puede aproximar por:

$$
\begin{aligned}
V\left(R_{p}\right)=\frac{1}{3(N+1)^{2}}\{ & \left.N\left(N^{2}+3 N+2\right)-[N p]\left([N p]^{2}+3[N p]-4\right)\right\} \\
& +3 p^{2}(N-1)\left(N^{2}-N[N p]+4\right) \\
- & \left.3 p\left(N^{3}+N^{2}+2 N-N[N p]^{2}-N[N p]+4[N p]\right)+6\right\} .
\end{aligned}
$$

Comunicaciones en Estadística, diciembre 2017, Vol. 10, No. 2 
La distribución de $R_{p}$ es simétrica alrededor de cero y la diferencia entre la distribución de $R_{p}$ (debidamente estandarizada) la distribución normal decrece con $N$, como se mostró en Babativa (2008), donde se calcularon las diferencias de Kolmogoroff entre la distribución exacta de $R_{p}$ y la distribución normal para $N$ entre 1 y 30. Por otra parte, los teoremas de Berry-Essen para sumas de variables aleatorias dependientes garantizan que dichas diferencias decrecen con $N$ (Hall \& Heyde (2014), sección 3.6). Lo anterior justifica el uso de la aproximación de la distribuciń de la estadística $R_{p}$ por la distribución normal para obtener los valores críticos de la prueba.

\section{Estudio de simulación y resultados}

Los resultados están divididos en dos partes: una que corresponde a la calibración del tamaño de las pruebas y otra al cálculo y comparación de las potencias empíricas, en ambos casos se simularon 3000 muestras de tamaño $N=30$.

Para la calibración se utilizó como distribución simétrica la normal de media cero y varianza $\sigma=0.3(0.1) 1{ }^{1}$

Para el cálculo de las potencias empíricas de las pruebas comparadas, las muestras se simularon de la distribución lognormal de parámetros $\mu$ y $\sigma(D L N(\mu, \sigma))$, $\mu=0(0.1) 0.6$ y $\sigma=0.3(0.1) 1$. Para la selección de la proporción de recorte, se calcularon las potencias para varios valores de $p$ observándose que las mejores potencias empíricas se obtienen para una proporción de recorte $p=0.80$ (Babativa $\&$ Corzo 2010) . Para cada valor de $\mu$ se obtienen ocho distribuciones cuyo grado de asimetría varía con los valores de $\sigma$ (Figura B.1) .

Se comparó la potencia empírica de la prueba propuesta con la de las siguientes pruebas:

1. Prueba de Cabilio \& Masaro (1996) que utiliza la estadística

$$
C M=\frac{\sqrt{N}(\bar{X}-\hat{\theta})}{s},
$$

donde $\bar{X}, \hat{\theta}$ y $s^{2}$ son la media, la mediana y la varianza muestral respectivamente.

2. Prueba de Mira (1999) basada en la estadística

$$
M=2(\bar{X}-\hat{\theta}),
$$

3. Prueba de Miao et al. (2006) que utiliza la estadística

$$
M G G=\frac{\sqrt{N}(\bar{X}-\hat{\theta})}{J},
$$

\footnotetext{
${ }^{1}$ La notación $a=b(c) d$ significa que $a$ varía de $b$ hasta $d$ con incrementos de $c$
} 
donde

$$
J=\frac{1}{2} \sqrt{\frac{\pi}{2}} \sum_{i=1}^{N}\left|X_{i}-\hat{\theta}\right| .
$$

4. Welch (1938) adaptada para la alternativa $K_{2}$ con mediana desconocida y estadística

$$
t R W=\frac{\bar{r}_{2}-\bar{r}_{1}}{\sqrt{\frac{s_{1}^{2}}{n_{1}}+\frac{s_{2}^{2}}{n_{2}}}}
$$

donde $r_{1 j}\left(j=1, \ldots, n_{1}\right)$ y $r_{2 k}\left(k=1, \ldots, n_{2}\right)$ denotan los rangos de las $n_{1}$ observaciones positivas y de las $n_{2}$ observaciones negativas respectivamente. Además, $\bar{r}_{1}, \bar{r}_{2}$ y $s_{1}^{2}, s_{2}^{2}$ denotan las medias y varianzas de $r_{1 j}$ y $r_{2 k}$ respectivamente.

En la tabla 1, ilustrada en la Figura 8, se verifica que en todos los casos las pruebas mantienen su tamaño alrededor del tamaño prefijado $\alpha=0.05$. Esto significa que desde el punto de vista empírico las pruebas comparadas son insesgadas.

Los cálculos de las potencias empíricas de las pruebas se encuentran en las tablas 2 a 8 . Para cada valor de $\mu$ se construyó una tabla en cuyas filas se encuentran las potencias empíricas de las pruebas comparadas para las siete distribuciones lognormales que genera el cambio de $\sigma$ (figura B.2). Observando todas las tablas por filas, es decir para cambios en los valores de $\sigma$, se nota que en todos los casos las potencias empíricas son no decrecientes.

También se observa que las potencias de cada prueba crecen con $\mu$, aspecto que se ve mirando las correspondientes potencias de cada prueba de tabla en tabla para el valor de $\sigma$. Así por ejemplo, para la prueba $R_{p}$ la potencia empírica para $\sigma=0.3$ y $\mu=0$ es 0.357 , para $\mu=0.1$ es 0.5897 , para $\mu=0.2$ es 0.7297 y así sucesivamente.

Finalmente, se observa que tanto con respecto a incrementos en la varianza como a incrementos en la media, la potencia empírica de la prueba $R_{p}$ es mayor que la de las demás pruebas comparadas. Esto se evidencia en la tabla 9 en la cual se ve claramente que en el aumento de $\mu=0$ a $\mu=0.1$ se presentan las mayores diferencias entre las potencias empíricas de la prueba $R_{p}$, y que estas van disminuyendo con el incremento de $\mu$.

\section{Conclusiones y discusión}

La prueba propuesta muestra considerables ventajas con respecto a las otras pruebas comparadas: es empíricamente insesgada y consistente, su potencia supera la de las otras pruebas comparadas, pero las diferencias son mayores especialmente para valores de $\sigma \leq 0.5$ y esto se cumple para todos los valores de $\mu$; consigue 
la máxima potencia más rápido que las otras pruebas en la medida en que $\mu$ va creciendo y las distribuciones muestreadas son más aplanadas.

En la tabla 9 se encuentran las diferencias en potencia para todos los valores de $\mu$ y $\sigma$ utilizados en la simulación. Se notan claramente las ventajas de la prueba propuesta para valores de $\mu$ y $\sigma$ pequeños y que las diferencias se van disminuyendo en la medida en que estos aumentan, al punto que para valores de $\sigma$ de 0.7 en adelante y cualquier valor de $\mu$ las diferencias son todas menores del $5 \%$. Lo anterior permite comprobar que las pruebas comparadas son localmente más potentes, con ventajas de la propuesta sobre las otras pruebas debido a que su potencia empírica crece más rápido, esto hace que la prueba sea recomendable para distribuciones más aplanadas.

La estadística de prueba propuesta utiliza solo el $20 \%$ de observaciones más grandes, por lo cual tolera cambios en el $80 \%$ de las observaciones pequeñas, antes de que se produzcan cambios en sus valores que afecten los resultados de las potencias empíricas. Adicionalmente, la mediana tolera hasta el $50 \%$ de cambios en observaciones mayores o menores lo cual hace plausible su uso para centrar las observaciones sin que se afecten los resultados.

Una pregunta natural es si se pueden encontrar valores de $p$ que produzcan mejores potencias de la prueba propuesta para muestras de distribuciones más aplanadas. También queda pendiente estudiar la potencia de la prueba propuesta en muestras de otras familias de distribuciones asimétricas para comprobar si la prueba propuesta conserva las propiedades que mostró para la distribución lognormal.

Recibido: 19 de Enero de 2017 Aceptado: 30 de Agosto de 2017

\section{Referencias}

Babativa, G. \& Corzo, J. (2010), 'Propuesta de una prueba de rachas recortada para la hipótesis de simetría', Revista Colombiana de Estadística 33(2), 251271.

Babativa, J. (2008), Propuesta de una prueba de rachas recortada para hipótesis de simetría, Tesis de Maestría, Universidad Nacional de Colombia, Facultad de Ciencias. Departamento de Estadística, Bogotá.

Cabilio, P. \& Masaro, J. (1996), 'A simple test of symmetry about an unknown median', The Canadian Journal of Statistics 24(3), 349-361.

Chatterjee, S. \& Sen, P. (1971), 'On Kolmogorov-Smirnov-Type Tests for Symmetry', Annals of the Institute of Statistical Mathematics 25(1), 287-299. 
Corzo, J. (1989), Verallgemeinerte Runtests for Lage- und Skalenalternativen, Tesis de doctorado, Universitat Dortmund, Fachbereich Statistik, Universitat Dortmund, .

Corzo, J. \& Babativa, G. (2013), 'A trimmed runs test for symmetry', Journal of Statistics Computation and Simulation 83(5), 984-991.

Hall, P. \& Heyde, C. C. (2014), Martingale limit theory and its application, Academic press.

McWilliams, P. (1990), 'A Distribution-Free Test for Symmetry Based on a Runs Statistic', Journal of the American Statistical Association 85(412), 11301133.

Miao, W., Gel, Y. \& Gastwirth, J. (2006), 'A new test of symmetry about an unknown median', Random Walk, Sequential Analysis and Related Topics A Festschrift in Honor of Yuan-Shih Chow. Eds.: Agnes Hsiung, Cun-Hui Zhang, and Zhiliang Ying, World Scientific Publishe pp. 1-19.

Mira, A. (1999), 'Distribution-free test for symmetry based on Bonferroni's measure', Journal of Applied Statistics 26(8), 959-972.

Modarres, R. \& Gastwirth (1996), 'A modified runs tes for symmetry', Statistics and probability $\mathbf{2 5}(5), 575-585$.

Modarres, R. \& Gastwirth, J. (1998), 'Hybrid test for the hypothesis of symmetry', Journal of Applied Statistics 25(6), 777-783.

Welch, B. L. (1938), 'The significance of the difference between two means when the population variances are unequal', Biometrika 29, 350-362. 


\section{A. Tablas}

Tabla 1: Calibración p-value para muestras de la distribución $N(0, \sigma)$. Fuente: elaboración propia.

\begin{tabular}{ccccccccc}
\hline & $\sigma=0.3$ & $\sigma=0.4$ & $\sigma=0.5$ & $\sigma=0.6$ & $\sigma=0.7$ & $\sigma=0.8$ & $\sigma=0.9$ & $\sigma=1$ \\
\hline$R_{p}$ & 0.0517 & 0.0537 & 0.0660 & 0.0553 & 0.0540 & 0.0563 & 0.0553 & 0.0563 \\
$M G G$ & 0.0390 & 0.0380 & 0.0487 & 0.0430 & 0.0403 & 0.0437 & 0.0397 & 0.0413 \\
$C M$ & 0.0377 & 0.0360 & 0.0460 & 0.0397 & 0.0377 & 0.0383 & 0.0363 & 0.0383 \\
$M$ & 0.0347 & 0.0363 & 0.0457 & 0.0360 & 0.0367 & 0.0367 & 0.0337 & 0.0387 \\
$t R W$ & 0.0563 & 0.0607 & 0.0670 & 0.0603 & 0.0623 & 0.0660 & 0.0597 & 0.0660 \\
\hline
\end{tabular}

Tabla 2: Potencias de las pruebas para $\operatorname{DLN}(0, \sigma)$. Fuente: elaboración propia.

\begin{tabular}{ccccccccc}
\hline & $\sigma=0.3$ & $\sigma=0.4$ & $\sigma=0.5$ & $\sigma=0.6$ & $\sigma=0.7$ & $\sigma=0.8$ & $\sigma=0.9$ & $\sigma=1$ \\
\hline$R_{p}$ & 0.3570 & 0.5177 & 0.6397 & 0.7503 & 0.8370 & 0.8993 & 0.9363 & 0.9597 \\
$M G G$ & 0.2697 & 0.4053 & 0.5277 & 0.6607 & 0.7563 & 0.8423 & 0.8977 & 0.9457 \\
$C M$ & 0.2330 & 0.3467 & 0.4480 & 0.5743 & 0.6783 & 0.7723 & 0.8203 & 0.8787 \\
$M$ & 0.2223 & 0.3357 & 0.4193 & 0.5320 & 0.6317 & 0.7220 & 0.7633 & 0.8167 \\
$t R W$ & 0.1917 & 0.2597 & 0.3013 & 0.3713 & 0.4367 & 0.5023 & 0.5287 & 0.5920 \\
\hline
\end{tabular}

Tabla 3: Potencias de las pruebas para $\operatorname{DLN}(0.1, \sigma)$. Fuente: elaboración propia.

\begin{tabular}{ccccccccc}
\hline & $\sigma=0.3$ & $\sigma=0.4$ & $\sigma=0.5$ & $\sigma=0.6$ & $\sigma=0.7$ & $\sigma=0.8$ & $\sigma=0.9$ & $\sigma=1$ \\
\hline$R_{p}$ & 0.5897 & 0.7707 & 0.8717 & 0.9367 & 0.9750 & 0.9880 & 0.9970 & 0.9993 \\
$M G G$ & 0.4633 & 0.6487 & 0.7823 & 0.8870 & 0.9437 & 0.9747 & 0.9903 & 0.9960 \\
$C M$ & 0.4037 & 0.5780 & 0.6987 & 0.8253 & 0.8957 & 0.9497 & 0.9703 & 0.9850 \\
$M$ & 0.3937 & 0.5603 & 0.6677 & 0.7873 & 0.8723 & 0.9230 & 0.9513 & 0.9623 \\
$t R W$ & 0.3557 & 0.4483 & 0.5113 & 0.6077 & 0.6727 & 0.7393 & 0.7823 & 0.8287 \\
\hline
\end{tabular}

Tabla 4: Potencias de las pruebas para $\operatorname{DLN}(0.2, \sigma)$. Fuente: elaboración propia.

\begin{tabular}{ccccccccc}
\hline & $\sigma=0.3$ & $\sigma=0.4$ & $\sigma=0.5$ & $\sigma=0.6$ & $\sigma=0.7$ & $\sigma=0.8$ & $\sigma=0.9$ & $\sigma=1$ \\
\hline$R_{p}$ & 0.7297 & 0.8787 & 0.9497 & 0.9827 & 0.9940 & 0.9980 & 1.0000 & 1.0000 \\
$M G G$ & 0.6027 & 0.7870 & 0.8903 & 0.9603 & 0.9827 & 0.9953 & 0.9997 & 0.9993 \\
$C M$ & 0.5313 & 0.7147 & 0.8340 & 0.9253 & 0.9633 & 0.9863 & 0.9933 & 0.9973 \\
$M$ & 0.5220 & 0.6887 & 0.8010 & 0.9033 & 0.9497 & 0.9767 & 0.9883 & 0.9910 \\
$t R W$ & 0.4750 & 0.5797 & 0.6497 & 0.7597 & 0.8117 & 0.8643 & 0.8923 & 0.9290 \\
\hline
\end{tabular}


Tabla 5: Potencias de las pruebas para $D L N(0.3, \sigma)$. Fuente: elaboración propia.

\begin{tabular}{ccccccccc} 
& $\sigma=0.3$ & $\sigma=0.4$ & $\sigma=0.5$ & $\sigma=0.6$ & $\sigma=0.7$ & $\sigma=0.8$ & $\sigma=0.9$ & $\sigma=1$ \\
\hline$R_{p}$ & 0.8310 & 0.9313 & 0.9827 & 0.9953 & 0.9987 & 1.0000 & 1.0000 & 1.0000 \\
$M G G$ & 0.7123 & 0.8720 & 0.9413 & 0.9850 & 0.9963 & 0.9997 & 1.0000 & 1.0000 \\
$C M$ & 0.6370 & 0.8107 & 0.8997 & 0.9663 & 0.9867 & 0.9960 & 0.9990 & 0.9997 \\
$M$ & 0.6273 & 0.7883 & 0.8807 & 0.9580 & 0.9783 & 0.9917 & 0.9983 & 0.9993 \\
$t R W$ & 0.5720 & 0.6793 & 0.7523 & 0.8513 & 0.8930 & 0.9317 & 0.9517 & 0.9737 \\
\hline
\end{tabular}

Tabla 6: Potencias de las pruebas para $D L N(0.4, \sigma)$. Fuente: elaboración propia.

\begin{tabular}{ccccccccc}
\hline & $\sigma=0.3$ & $\sigma=0.4$ & $\sigma=0.5$ & $\sigma=0.6$ & $\sigma=0.7$ & $\sigma=0.8$ & $\sigma=0.9$ & $\sigma=1$ \\
\hline$R_{p}$ & 0.8893 & 0.9640 & 0.9930 & 0.9987 & 1.0000 & 1.0000 & 1.0000 & 1.0000 \\
$M G G$ & 0.7840 & 0.9217 & 0.9700 & 0.9933 & 0.9990 & 1.0000 & 1.0000 & 1.0000 \\
$C M$ & 0.7147 & 0.8737 & 0.9450 & 0.9827 & 0.9950 & 0.9987 & 1.0000 & 1.0000 \\
$M$ & 0.7090 & 0.8550 & 0.9313 & 0.9780 & 0.9907 & 0.9960 & 1.0000 & 0.9997 \\
$t R W$ & 0.6527 & 0.7540 & 0.8280 & 0.9003 & 0.9360 & 0.9677 & 0.9807 & 0.9910 \\
\hline
\end{tabular}

Tabla 7: Potencias de las pruebas para $D L N(0.5, \sigma)$. Fuente: elaboración propia.

\begin{tabular}{ccccccccc}
\hline & $\sigma=0.3$ & $\sigma=0.4$ & $\sigma=0.5$ & $\sigma=0.6$ & $\sigma=0.7$ & $\sigma=0.8$ & $\sigma=0.9$ & $\sigma=1$ \\
\hline$R_{p}$ & 0.9267 & 0.9807 & 0.9980 & 1.0000 & 1.0000 & 1.0000 & 1.0000 & 1.0000 \\
$M G G$ & 0.8423 & 0.9510 & 0.9850 & 0.9987 & 0.9993 & 1.0000 & 1.0000 & 1.0000 \\
$C M$ & 0.7787 & 0.9170 & 0.9700 & 0.9937 & 0.9980 & 1.0000 & 1.0000 & 1.0000 \\
$M$ & 0.7693 & 0.8997 & 0.9600 & 0.9917 & 0.9963 & 0.9987 & 1.0000 & 1.0000 \\
$t R W$ & 0.7203 & 0.8197 & 0.8763 & 0.9373 & 0.9627 & 0.9843 & 0.9920 & 0.9967 \\
\hline
\end{tabular}

Tabla 8: Potencias de las pruebas para $D L N(0.6, \sigma)$. Fuente: elaboración propia.

\begin{tabular}{ccccccccc}
\hline & $\sigma=0.3$ & $\sigma=0.4$ & $\sigma=0.5$ & $\sigma=0.6$ & $\sigma=0.7$ & $\sigma=0.8$ & $\sigma=0.9$ & $\sigma=1$ \\
\hline$R_{p}$ & 0.9527 & 0.9877 & 0.9987 & 1.0000 & 1.0000 & 1.0000 & 1.0000 & 1.0000 \\
$M G G$ & 0.8893 & 0.9690 & 0.9920 & 1.0000 & 0.9997 & 1.0000 & 1.0000 & 1.0000 \\
$C M$ & 0.8300 & 0.9437 & 0.9833 & 0.9983 & 0.9987 & 1.0000 & 1.0000 & 1.0000 \\
$M$ & 0.8247 & 0.9330 & 0.9783 & 0.9967 & 0.9977 & 0.9993 & 1.0000 & 1.0000 \\
$t R W$ & 0.7710 & 0.8607 & 0.9117 & 0.9610 & 0.9780 & 0.9900 & 0.9960 & 0.9983 \\
\hline
\end{tabular}

Tabla 9: Diferencias. Fuente: elaboración propia.

\begin{tabular}{ccccccccc}
\hline Dif. de $\mu$ & $\sigma=0.3$ & $\sigma=0.4$ & $\sigma=0.5$ & $\sigma=0.6$ & $\sigma=0.7$ & $\sigma=0.8$ & $\sigma=0.9$ & $\sigma=1$ \\
\hline de 0.1 a 0.2 & 0.1400 & 0.1080 & 0.0780 & 0.0460 & 0.0190 & 0.0100 & 0.0030 & 0.0007 \\
de 0.2 a 0.3 & 0.1013 & 0.0526 & 0.0330 & 0.0126 & 0.0047 & 0.0020 & 0 & 0 \\
de 0.3 a 0.4 & 0.0583 & 0.0327 & 0.0103 & 0.0034 & 0.0013 & 0 & 0 & 0 \\
de 0.4 a 0.5 & 0.0374 & 0.0167 & 0.0050 & 0.0013 & 0 & 0 & 0 & 0 \\
de 0.5 a 0.6 & 0.0260 & 0.0070 & 0.0007 & 0 & 0 & 0 & 0 & 0 \\
\hline
\end{tabular}




\section{B. Figuras}

\section{B.1. Función de densidad $D L N(\mu, \sigma)$}

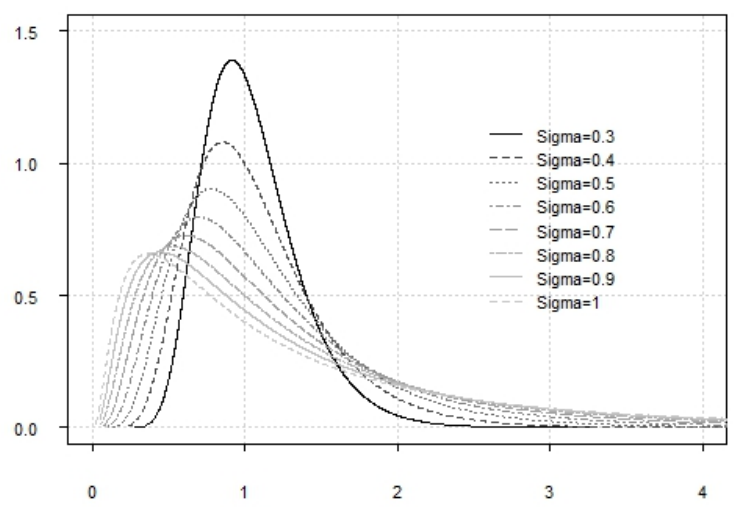

Figura 1: Función de densidad de la $D L N(0, \sigma)$. Fuente: elaboración propia.

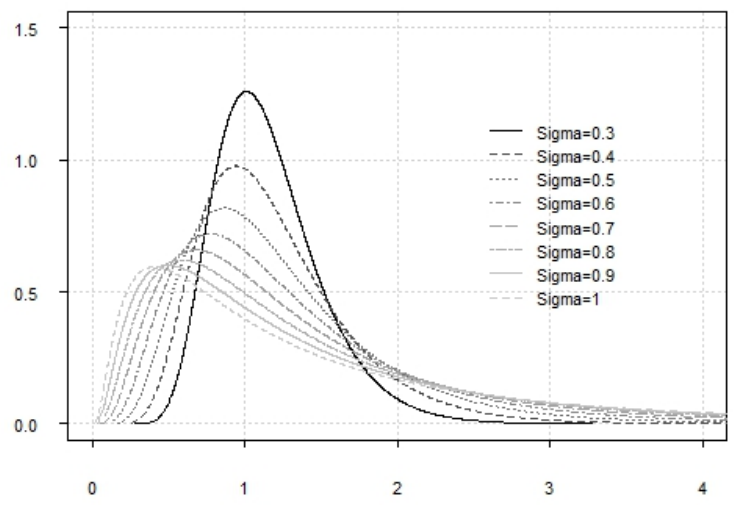

Figura 2: Función de densidad de la $\operatorname{DLN}(0.1, \sigma)$. Fuente: elaboración propia. 


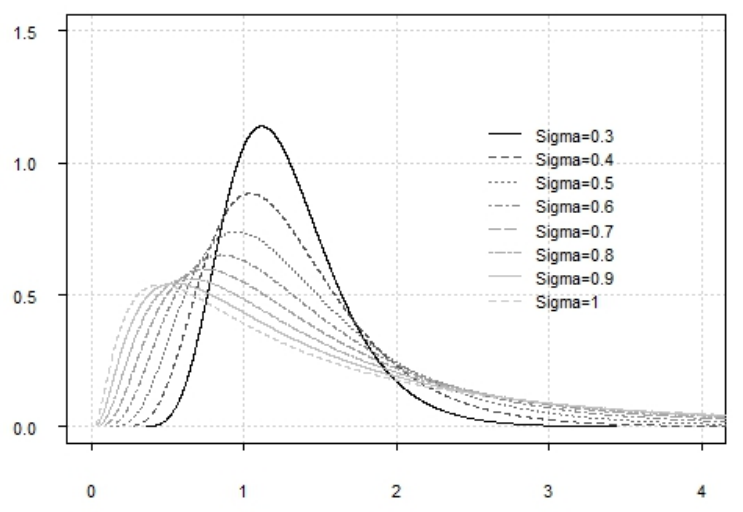

Figura 3: Función de densidad de la $\operatorname{DLN}(0.2, \sigma)$. Fuente: elaboración propia.

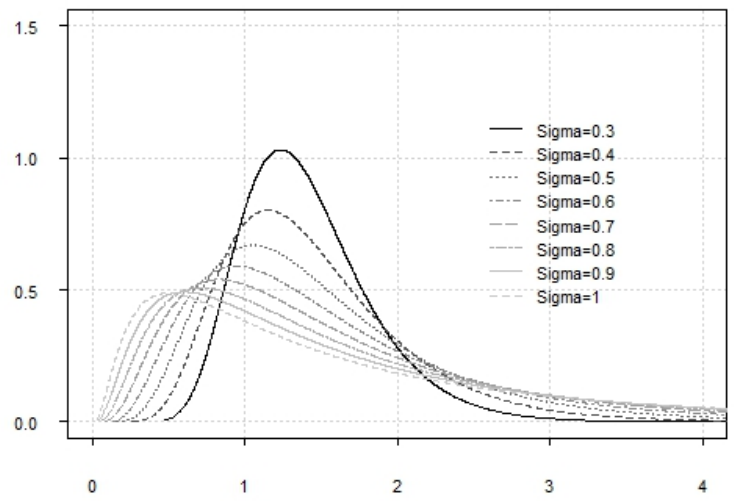

Figura 4: Función de densidad de la $\operatorname{DLN}(0.3, \sigma)$. Fuente: elaboración propia.

Comunicaciones en Estadística, diciembre 2017, Vol. 10, No. 2 


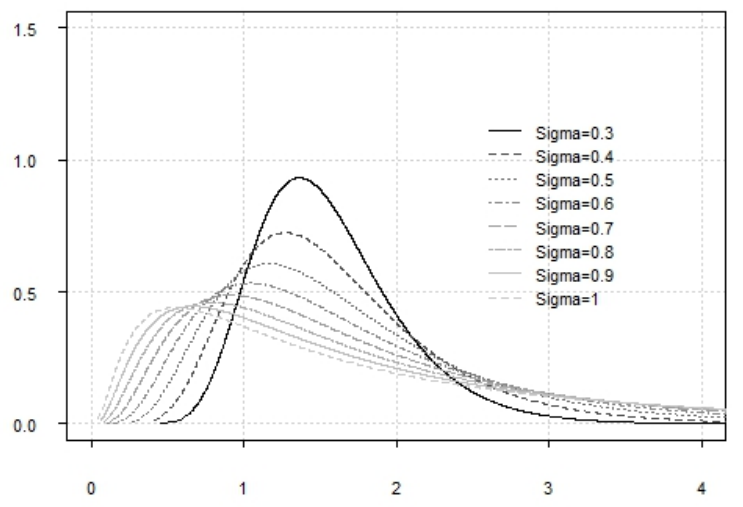

Figura 5: Función de densidad de la $\operatorname{DLN}(0.4, \sigma)$. Fuente: elaboración propia.

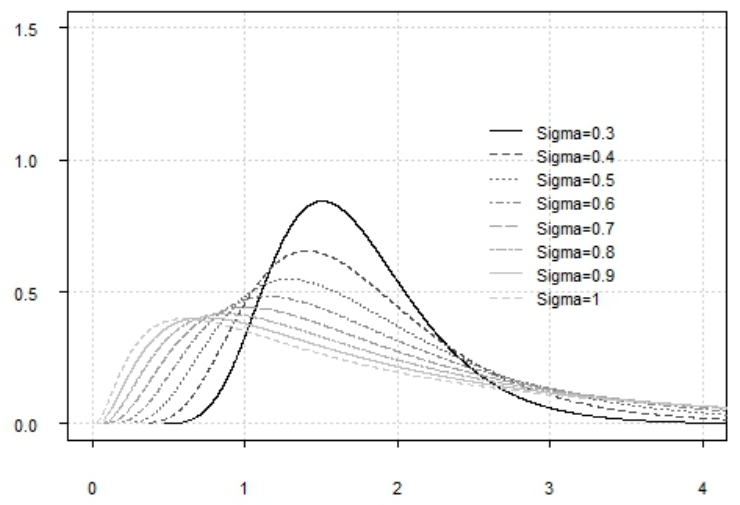

Figura 6: Función de densidad de la $\operatorname{DLN}(0.5, \sigma)$. Fuente: elaboración propia.

Comunicaciones en Estadística, diciembre 2017, Vol. 10, No. 2 


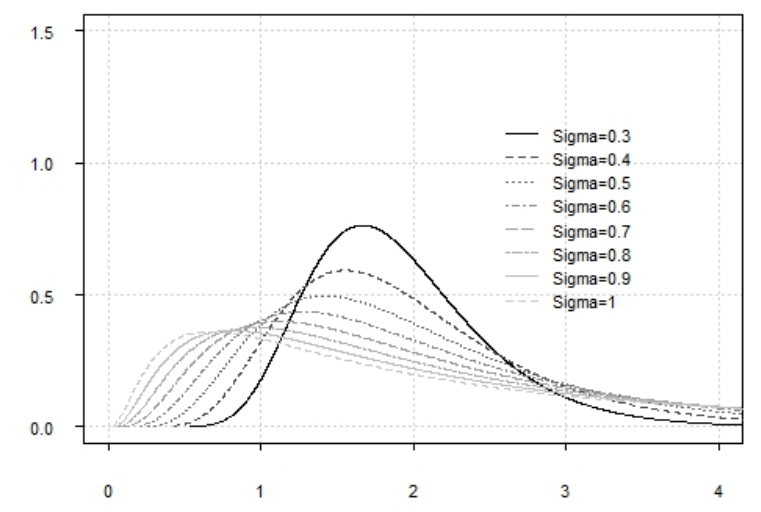

Figura 7: Función de densidad de la $\operatorname{DLN}(0.6, \sigma)$. Fuente: elaboración propia. 


\section{B.2. Potencias de las pruebas $D L N(\mu, \sigma)$}

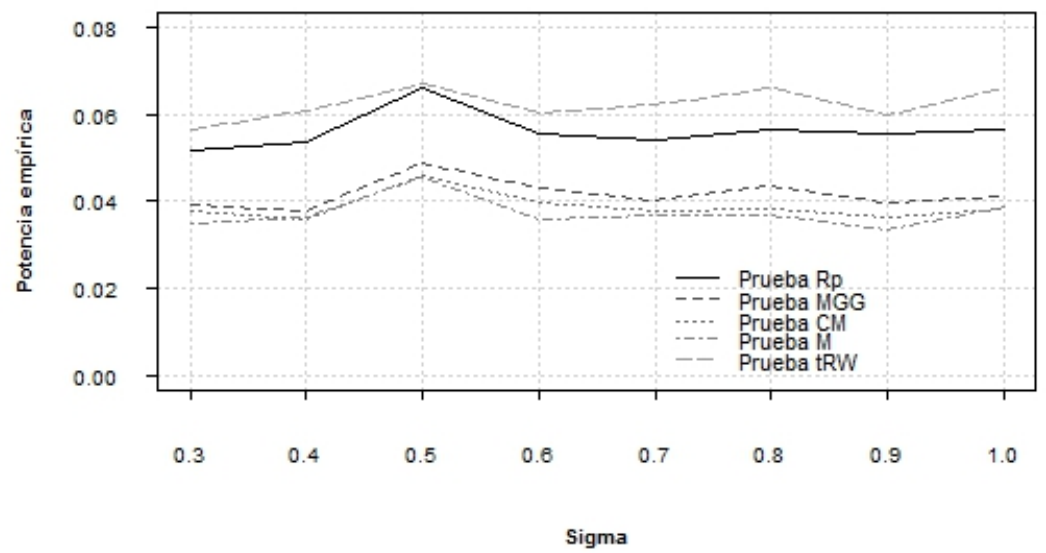

Figura 8: Calibración p-value muestras distribución $N(0, \sigma)$. Fuente: elaboración propia.

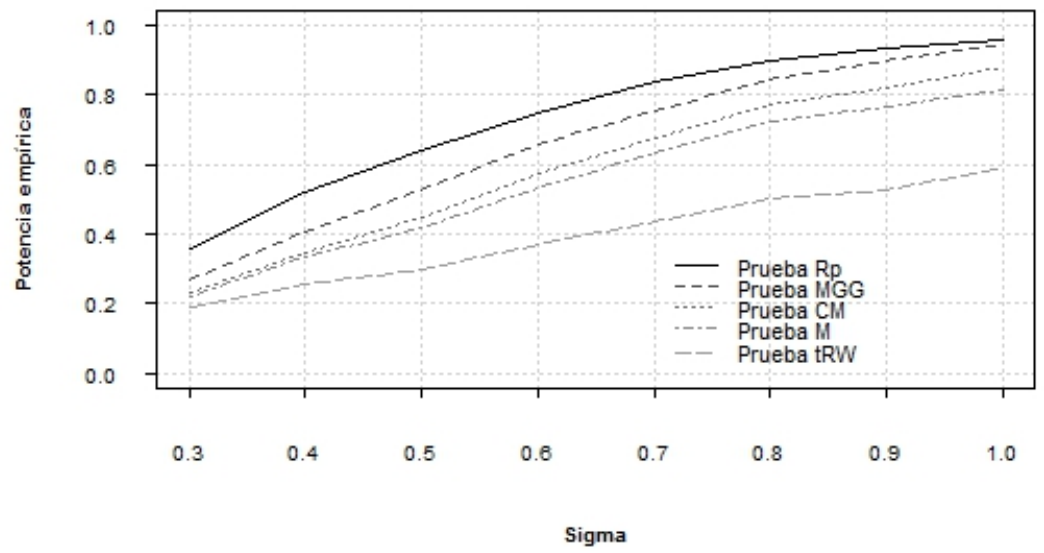

Figura 9: Potencias de las pruebas para $\operatorname{DLN}(0, \sigma)$. Fuente: elaboración propia.

Comunicaciones en Estadística, diciembre 2017, Vol. 10, No. 2 


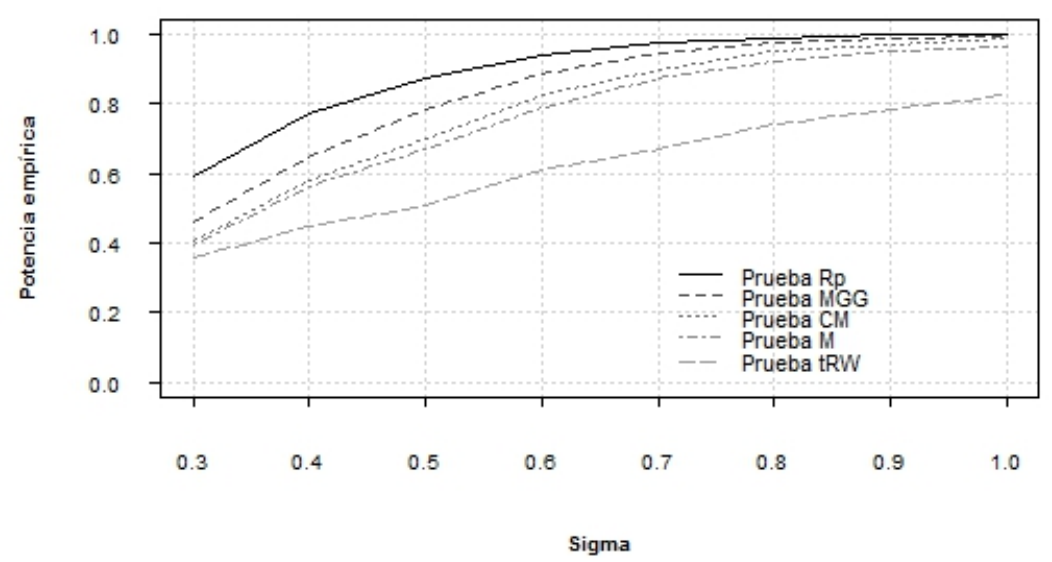

Figura 10: Potencias de las pruebas para $D L N(0.1, \sigma)$. Fuente: elaboración propia.

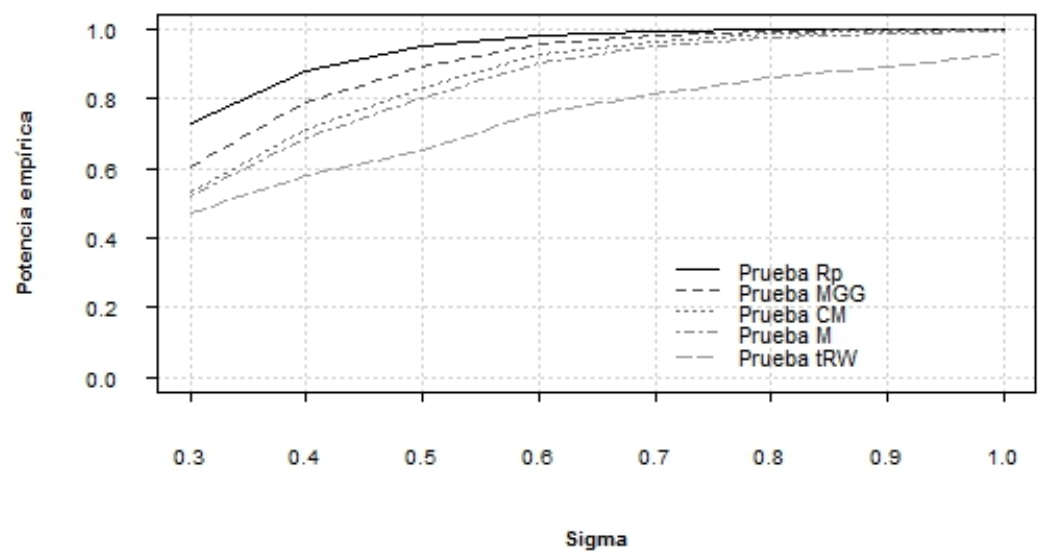

Figura 11: Potencias de las pruebas para $\operatorname{DLN}(0.2, \sigma)$. Fuente: elaboración propia.

Comunicaciones en Estadística, diciembre 2017, Vol. 10, No. 2 


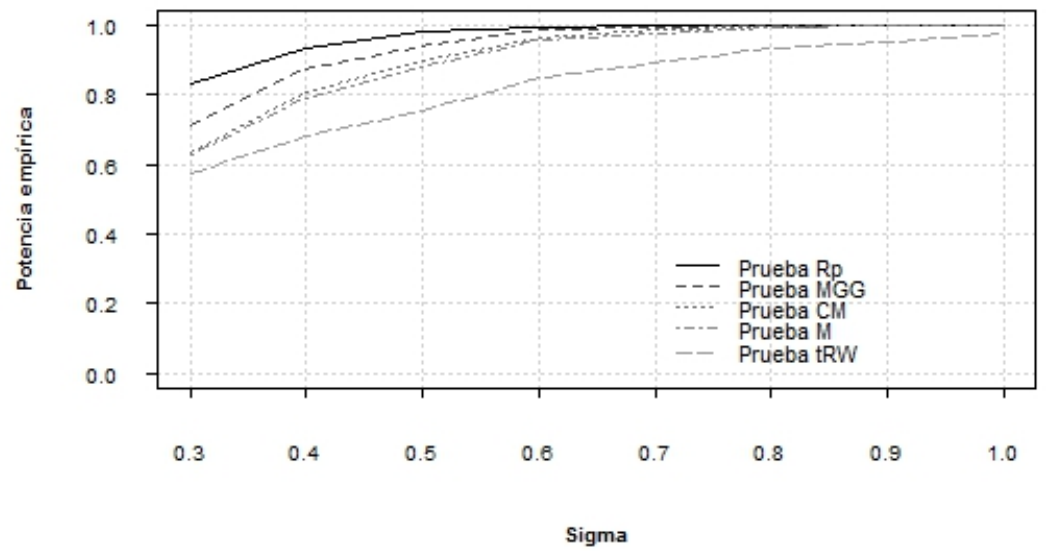

Figura 12: Potencias de las pruebas para $\operatorname{DLN}(0.3, \sigma)$. Fuente: elaboración propia.

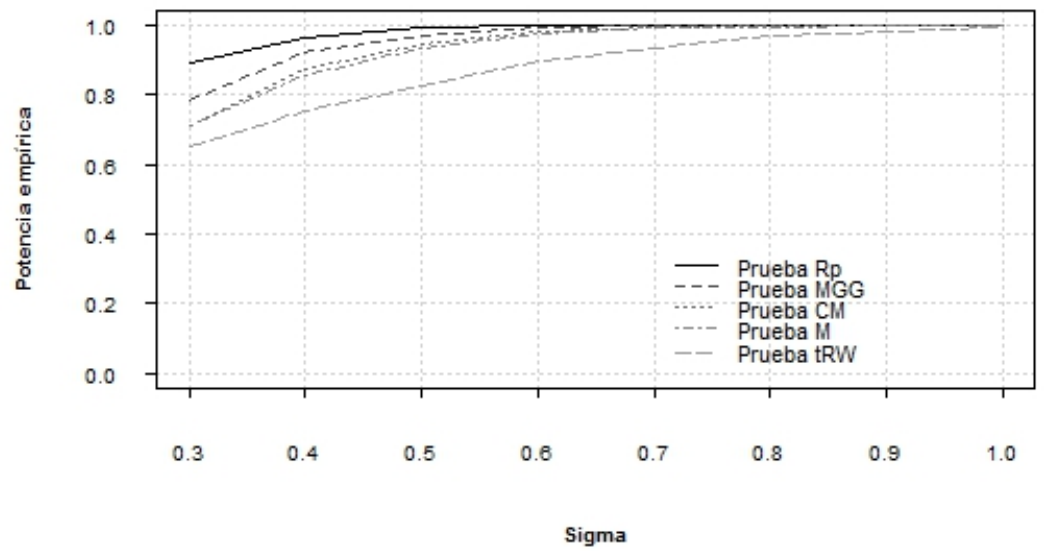

Figura 13: potencias de las pruebas para $\operatorname{DLN}(0.4, \sigma)$. Fuente: elaboración propia.

Comunicaciones en Estadística, diciembre 2017, Vol. 10, No. 2 


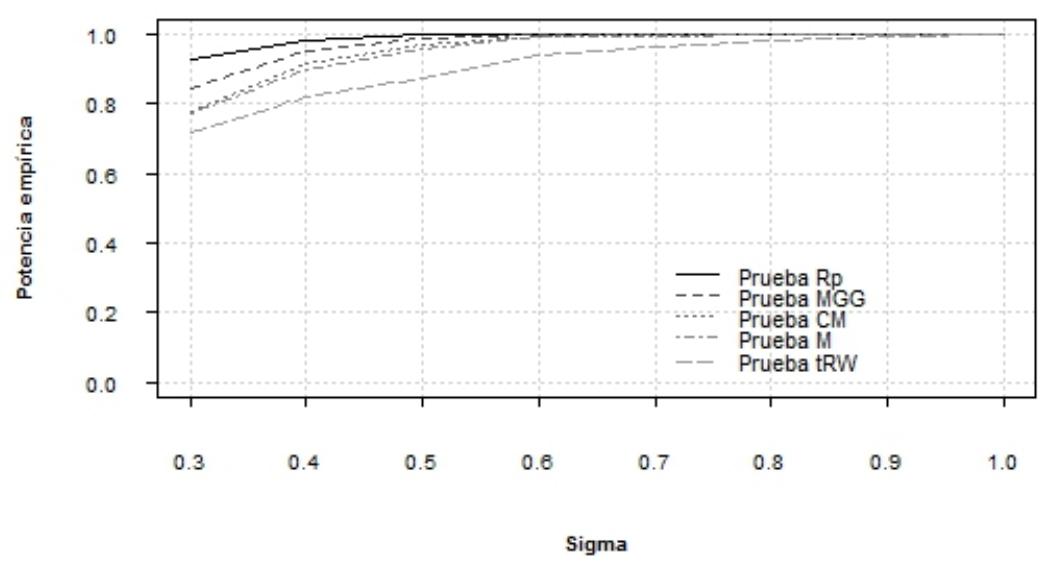

Figura 14: Potencias de las pruebas para $\operatorname{DLN}(0.5, \sigma)$. Fuente: elaboración propia.

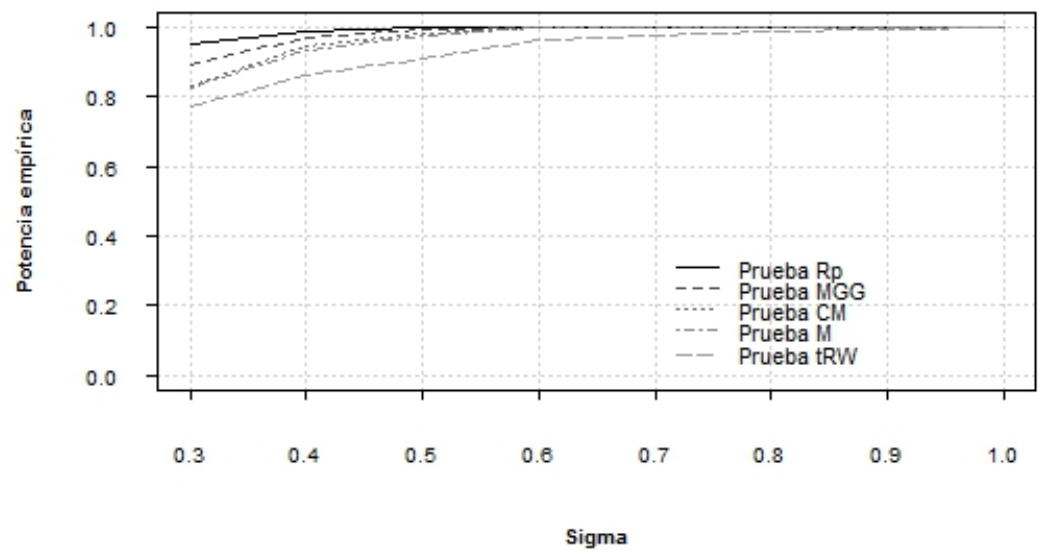

Figura 15: Potencias de las pruebas para $\operatorname{DLN}(0.6, \sigma)$. Fuente: elaboración propia.

Comunicaciones en Estadística, diciembre 2017, Vol. 10, No. 2 